\title{
Exploration and Practice of Online and Offline Blending Teaching Mode Based on "SPOC + DingTalk"
}

\author{
Yinyin $\mathrm{Xu}^{*}$ \\ Ningbo University of Technology, Ningbo 315211, Zhejiang Province, China. E-mail: 695854894@qq.com
}

\begin{abstract}
This paper analyzes the characteristics of the blending teaching mode of SPOC (Small Private Online Course) and DingTalk. From three major teaching links, the design and development of learning resources, the evaluation and suggestions of teaching activities, the online and offline blending teaching model of "SPOC + DingTalk" is discussed to provide implementation suggestions for teaching management and practical subjects including teachers.
\end{abstract}

Keywords: SPOC; DingTalk; Online and Offline; Blending Teaching

\section{Introduction}

The 21 st century is an era with rapid development of the Internet, and important breakthroughs in science and technology often have an impact on the development of education to a certain extent. Based on the development of the "SPOC + DingTalk" online and offline blending teaching model, the teaching methods are more technical, the teaching concepts are more humanized, and the teaching content is diversified. In higher education, the indispensable element is online teaching, whose outstanding advantages attract both teachers and the majority of students. However, offline education is a core component of the traditional classroom teaching model. Only a perfect combination of online and offline teaching models can better serve students, so that students can not only grasp knowledge but also follow the trend of the times.

\section{Overview of "SPOC + DingTalk" teaching mode}

SPOC (Small Private Online Course) is more refined than MOOC (Massive Open Online Courses) ${ }^{[1]}$. The concept of SPOC was proposed by Professor Armando Fox of the University of California, Berkeley in 2013. Specifically, "Small" refers to the scale of tens to hundreds of people; "Private" refers to the setting of restrictive quasi-entry conditions. SPOC makes full use of the advantages of MOOC. It is a hybrid teaching model that combines online education with offline physical teaching. It is considered to be a more suitable curriculum model for students in school ${ }^{[2]}$. The SPOC platform supports a hybrid teaching model, which has many advantages: the teaching method is changed to "student-centered" instead of "teacher-centered" in the traditional teaching model, that is to say, the focus is on students; students can personally study and actively communicate with teachers, and receive personalized teaching according to their characteristics; with the help of network teaching platform, teachers, students and teaching resources can be effectively integrated to establish a diversified blending teaching model; teachers' management on students is more intelligent and efficient. With the help of data analysis on the online platform, teachers can effectively understand the time, progress and effects of student learning, and be fully familiar with the students' learning condition before, during

This is an open-access article distributed under the terms of the Creative Commons Attribution Non-Commercial License

(http://creativecommons.org/licenses/by-nc/4.0/), which permits unrestricted non-commercial use, distribution, and reproduction in any medium, provided the original work is properly cited. 
and after class. Teachers can adjust the progress of classroom teaching methods and content according to the actual situation. By means of SPOC blending teaching, students use Internet online resources for independent learning and cooperate in groups, while teachers help to solve their doubts and carry out personality counseling ${ }^{[3]}$.

DingTalk is a multi-terminal platform for free communication and collaboration created by Alibaba Group for Chinese enterprises and institutions. Among them, there are online classrooms dedicated to online teaching and interactive learning services for teachers and students, covering functions such as online communication, online organization and DingTalk education ${ }^{[4]}$. Webcast is one of the functional modules under DingTalk Education. The main advantages are the diversity of teaching content. During the live broadcast, teachers can not only use traditional courseware to teach, but also obtain online multimedia learning resources from the Internet at any time, so that the diversity of teaching resources has been greatly improved. Meanwhile, classroom interaction has been greatly improved. During the live broadcast, students can apply for connect microphone. After the teacher accepts the student's application, discussion and communication between teachers and students can be realized. Through live broadcast playback, students can repeatedly study videos according to their learning situation to fully grasp knowledge and improve learning efficiency.

\section{Blending teaching practice based on "SPOC + DingTalk"}

\subsection{Three links in teaching}

\subsubsection{Before class}

Students need to make the following preparations: preparing the hardware (mobile phone or computer) to keep the network signal normal; debugging the volume of the device to ensure the quality of the hardware environment for the class; according to the information posted on the DingTalk platform, pre-learning with learning purpose, learning content, and learning objectives, thinking about the questions raised by teachers before the class or completing the test published. During the pre-learning process, in case of doubt, students can ask the teacher for help ${ }^{[5]}$. Teachers need to make technical preparations for software applications before class: to complete online teaching with quality and quantity, teachers must carefully study the platform usage guidelines provided by SPOC teaching platform, DingTalk, etc., consult platform customer service or experienced teachers, and continue to explore and master the operation of the teaching software, so as to quickly solve the abnormal situations. Considering the students' learning situation, teachers publish the learning tasks, questions and tests, and timely adjust the key and difficult points and teaching content in the course, so as to fully grasp the students' pre-class learning situation.

\subsubsection{In class}

After the preview, the teacher answers the important and difficult points of knowledge, and focus on the explanation of the knowledge points in the class. After solving the learning confusion of the students, the teacher should guide the students to reflect and summarize the knowledge points step by step, and ensure students to truly master and comprehend the knowledge. The teaching methods can be flexible and changeable. When the student initiates the connect microphone application, the teacher should contact the student in time and obtain the student's learning situation in time.

\subsubsection{After class}

Students need to digest and absorb the knowledge they have learned. Through project-based exercises, they can better review and consolidate the knowledge they have learned to achieve the effect of learning. In this session, with the help of online assignments, online submissions, and online comment assignments in the DingTalk platform, teachers can receive feedback from students in time to better understand students' mastery of learning. Students can put forward opinions or suggestions to teachers, and teachers should reflect and summarize students' opinions and make corrections in the subsequent teaching process to achieve the purpose of teaching. 


\subsection{Design and development of SPOC blending teaching resources}

In the initial stage of resource learning, resources can be divided into online resources and offline resources. As for the online resources, the existing high-quality learning resources like MOOC videos, quality courses and open courses can be applied to SPOC blending teaching. Teachers can also make teaching videos based on teaching needs to achieve teaching goals and improve teaching efficiency. Offline learning resources are mainly teaching materials required for classroom activities prepared by teachers ${ }^{[6]}$.

In the medium-term stage of resource learning, online resource learning should be organized in units of knowledge points. Each learning video should be between 10 and 15 minutes. The content should be focused and concise. The main activities in the offline classroom should be discussion and interaction. The learning time is more acceptable to students within 60 minutes.

In the later stage of resource learning, online learning is mainly based on supplement content, which is sent to students in the form of teaching videos. The types of homework are mainly objective questions such as judgment questions and multiple choice questions, so that teachers can quickly understand students' mastery of knowledge points. Students can conduct exploratory learning through offline discussions. Heuristic education can stimulate students' learning initiative.

\section{Evaluation of the blending teaching model based on "SPOC + DingTalk"}

Under the online and offline blending teaching mode, teachers and students need to change their concepts, follow the trend of the times, and actively adapt to the reform and innovation of the new teaching mode ${ }^{[7]}$. Through online learning, students learn with questions in the classroom. The traditional teacher-based classroom in the past has been gradually transformed into a student-based classroom, which improves the students' initiative in learning. Under the blending teaching model, students have a stronger sense of participation and more freedom in personal expression, thus markedly promoting participation. Online discussions and answering questions make students bolder to raise their own confusion. They can ask questions at any time, and teachers can answer questions at any time, and learning efficiency has gradually increased. The "SPOC + DingTalk" teaching model is a product of adapting to the times. It combines the advantages of small-scale restrictive online courses in SPOC and DingTalk live broadcast. This model is an innovation and a challenge that requires a high degree of cooperation between students and teachers. As a supporting platform for online teaching, DingTalk Teaching Platform is also an effective supporting system for auxiliary teaching in many colleges and universities. It accelerates the transformation of traditional concepts. The teaching mode of "SPOC + DingTalk" enriches the teaching content of the school and improves the quality of teaching and effectiveness. Technology provides a new teaching method for teaching. Internet is a good teaching platform that brings convenience to teaching. The rapid development of the network platform is supported by information technology and network technology. Education must adapt to mobile learning, universal learning and lifelong learning, which is determined by learning needs ${ }^{[8]}$.

But how to make good use of the advantages brought by these Internet requires further explore and practice as there are still some problems of theses teaching platforms due to insufficient research and development time and immature technology. The communication tools, teaching materials, and teaching interactions of online education are limited to the network with extremely high dependence. After the online education is disconnected from the network, the transmission of learning materials and documents will be interrupted, so as exchange and discussion. Therefore, how to perfectly combine online and offline blending teaching in the teaching process is a major problem that teachers should deal with. It is necessary for teachers to guide students correctly and connect with actual teaching content.

\section{Suggestions on the blending teaching modle based on "SPOC + DingTalk"}

In the blending teaching mode of "SPOC + DingTalk", the workload of teachers has increased significantly. 
Teachers need to build a class before class, familiarize themselves with the operation of the software in advance, ensure the quality of the class, transfer teaching resources, arrange for the release of pre-class tasks, and carefully prepare the lesson ${ }^{[9]}$. They should also organize students to carry out discussion and interaction, and answer questions raised by students after class. Teachers should strengthen the exploration of the "SPOC + DingTalk" teaching model. Online teaching is not simply to move traditional classrooms online, but to redesign courses, restructure teaching organizations, collect teaching resources through multiple channels, and use teaching platforms to develop online teaching. Teachers should change their view, take students as the center, do a good job of guiding students, and effectively connect online and offline to improve teaching quality.

\section{Conclusion}

SPOC upholds the advanced teaching design concept of MOOC, with free and high-quality curriculum resources, and the concept of open sharing, which can break the standardized and controlled single-line transmission classroom and promote the innovation of college education model ${ }^{[10]}$. The blending teaching based on "SPOC + DingTalk" can integrate online teaching and face-to-face traditional classroom teaching organically to optimize the teaching effect. At the same time, the application of SPOC curriculum resources in college teaching requires more researchers and practitioners to join, and it needs to be constantly tested and improved in practice.

\section{References}

1. Wu Q, Zeng X. Research on the application of online and offline blended teaching model in software technology courses of higher vocational colleges (in Chinese). Computer Products and Circulation 2020; (7): 151-152.

2. Zhang X. A case study of English teaching in vocational colleges based on SPOC hybrid teaching model (in Chinese). Wencun Yuekan 2017; (20): 83-84.

3. Meng J. A study on blended English teaching model in higher vocational colleges based on SPOC (in Chinese). Education and Teaching Forum 2020; (11): 350-351.

4. Wang D. Experience of making and using PPT of webcast course in higher vocational colleges: Taking DingTalk teaching platform as an example (in Chinese). South Agricultural Machinery 2020; 51(9): 195.

5. Zhang X, Jia C, Li H. The research and practice of hybrid teaching model of six auxiliary online courses: a case study of medium inorganic chemistry course based on Rain Classroom + DingTalk live streaming (in Chinese). University Chemistry 2020; 5(28): 1-8.

6. Liu F. Research on the design of mixed teaching mode of data structure course based on SPOC (in Chinese). Indusrty and Information Technology Education 2020; (4): 15-18.

7. Tang Y. Discussion on computer basic course reform in higher vocational colleges based on SPOC hybrid teaching model. Technology Wind 2020; (11): 83.

8. Liu Y, Hu R, Lou T, et al. The practice of online and offline blended teaching mode based on super star learning channel in the teaching of neurorehabilitation. Western China Quality Education 2020; 6(9): 127-128.

9. Fan J. Research on the influencing factors and promotion strategies of blended teaching in higher vocational colleges based on SPOC (in Chinese). The Theory and Practice of Innovation and Entrepreneurship 2020; 3(3): 57-58.

10. Liu Y. Research on hybrid teaching mode of system engineering introduction based on SPOC (in Chinese). Contemporary Education Research and Teaching Practice 2020; (4): 29-30. 